\title{
Avaliação da Inibição do Eixo Neuroendócrino com Contraceptivo Oral de Baixa Dosagem
}

Evaluation of Neuroendocrine Axis Inhibition with a Low-Dose Oral Contraceptive

Dalton Ferreira, Sebastião Freitas de Medeiros

\section{RESUM0}

Objetivo: avaliar as variações dos hormônios foliculo-estimulante (FSH) e luteinizante (LH) na semana de pausa em usuárias de anticoncepcional hormonal oral contendo $20 \mu \mathrm{g}$ de etinilestradiol combinado com $75 \mathrm{mg}$ de gestodene.

Métodos: foram incluidas 31 mulheres, com idade entre 17 e 36 anos, média de 24,5 anos; $19 \%$ eram adolescentes. FSH, LH, prolactina (PRL) e estradiol $\left(E_{2}\right)$ foram medidos por imunoquimioluminescência em um dos últimos quatro dias de ingestão do comprimido de uma cartela de 21 e no $7^{\circ}$ dia de pausa entre duas cartelas. Os parâmetros hormonais foram comparados pelo teste t de Student para amostras pareadas. Fez-se correlação entre os níveis de hormônios e dados antropométricos por regressão linear. Valores de $p \leq 0,05$ foram considerados significantes.

Resultados: setenta e um porcento das mulheres usavam o anticoncepcional pela primeira vez. As concentrações de FSH aumentaram de 1,3 $\mathrm{mUI} / \mathrm{ml}$ nos últimos dias da cartela para $5,7 \mathrm{mUI} / \mathrm{ml} \mathrm{no} 7^{\circ}$ dia de pausa. O LH aumentou de 0,8 $\mathrm{mUI} / \mathrm{ml}$ para 4,3 $\mathrm{mUI} / \mathrm{ml}$ neste mesmo período. $O$ aumento de $E_{2}$ foi de 20,2 para $28,0 \mathrm{pg} / \mathrm{ml}$. Os níveis de $P R L$ diminuíram de 12,4 para $10,2 \mathrm{ng} / \mathrm{ml}$. Não houve associação entre as variações das gonadotrofinas com parâmetros antropométricos em mulheres com índice de massa corpórea $\leq 25 \mathrm{~kg} / \mathrm{m}^{2}$.

Conclusão: a concentração sérica das gonadotrofinas nos últimos dias da cartela do anticoncepcional testado está intensamente suprimida, havendo incremento de 3 a 4 vezes nos dias de intervalo de uso.

PALAVRAS-CHAVE: Anticoncepção hormonal oral. Prolactina. Gonadotrofinas.

\section{Introdução}

Os anticoncepcionais hormonais orais (ACHO), comercializados a partir dos anos 60, sofreram redução na dose do componente estrogênico de 50 para $20 \mu \mathrm{g}$, ou mesmo $15 \mu \mathrm{g}$, em cada comprimido. O componente progestagênico, intensamente pesquisado, evoluiu com a síntese de novos compostos, quase desprovidos de atividade

Departamento de Ginecologia e Obstetrícia, Faculdade de Ciências Médicas, Universidade Federal de Mato Grosso

Correspondência:

Sebastião Freitas de Medeiros

Av. Marechal Deodoro, 1055/1302

78005-101 - Cuiabá - MT

Fone: (65) 322-7342

Fax: (65) 612-9090 androgênica. Os ACHO atuais são ingeridos durante 21-24 dias, seguindo-se intervalo livre de 47 dias antes do reinício. A pílula exerce seus efeitos principalmente pelo bloqueio parcial da foliculogênese e da inibição do pico de gonadotrofinas. A ação moduladora gonadotrópica/ antigonadotrópica dos estrogênios é conhecida há quase oito décadas, sendo este efeito dose e tempo-dependente ${ }^{1}$.

No hipotálamo, o estrogênio exerce retroalimentação negativa sobre o sistema neuronal responsável pela síntese do hormônio liberador de gonadotrofinas e, na hipófise, favorece a sintese e o armazenamento das gonadotrofinas, mas inibe sua liberação pelo gonadotropo ${ }^{2}$. O estrogênio mantém ainda a estabilidade endometrial e, pelo aumento da concentração dos receptores progestagênicos, potencializa a ação destes compostos ${ }^{3}$. O 
estrogênio é o principal responsável pela diminuição dos níveis de hormônio folículo-estimulante (FSH) e o componente progestagênico exerce bloqueio preferencial da secreção do hormônio luteinizante $(\mathrm{LH})^{4}$. No hipotálamo o progestagênio inibe a secreção de gonadotrofinas, alterando sua pulsatilidade. Na hipófise, tem efeito bifásico, sendo capaz tanto de inibir como, quando em baixas concentrações, estimular a liberação de $\mathrm{LH}^{5}$. Além da ação sobre o eixo hipotálamo-hipofisário, o ACHO torna o muco cervical espesso e inadequado para a penetração e sobrevivência do espermatozóide, e promove atrofia e diminui a secreção de glicogênio no endométrio. Nas tubas o ACHO altera a motilidade, dependendo da concentração relativa de estrogênio no preparado utilizado ${ }^{6}$.

As modificações dos níveis das gonadotrofinas na pausa entre duas cartelas de pílula anticoncepcional requerem maior investigação, principalmente aquelas decorrentes do uso das novas formulações contendo etinilestradiol em baixas doses e novos progestagênios. Este estudo tem como proposta avaliar a função hipotálamo-hipofisária das mulheres usuárias de um ACHO contendo $20 \mu \mathrm{g}$ de etinilestradiol associado a $75 \mu \mathrm{g}$ de gestodeno, analisando os niveis de gonadotrofinas, prolactina (PRL) e estradiol no término e reinício da seqüência de 21 comprimidos. Especificamente, avalia o grau de atenuação e recuperação dos níveis de FSH e LH e as modificações induzidas sobre a PRL no término do ciclo de pílulas.

\section{Pacientes e Métodos}

Este estudo coorte, analítico, inclui 31 voluntárias atendidas no ambulatório de Ginecologia e Obstetrícia do Hospital Universitário Júlio Muller, procurando orientação para anticoncepção. Os critérios de inclusão foram o desejo de anticoncepção, ciclos menstruais com intervalos entre 26 e 34 dias e a quiescência em assinar o termo de consentimento livre e esclarecido. Foram excluídas as pacientes com contra-indicação para o uso da combinação estrogênio-progestagênio, tabagistas, diabéticas, usuárias de drogas, préobesas e obesas e, pela possibilidade de atenuação das gonadotrofinas nestas condições, as atletas e bailarinas.

Todas foram submetidas a avaliação clínico-ginecológica no início e no final do estudo, por um dos investigadores. No ciclo de seguimento em uso da pílula, as participantes foram orientadas para o registro de qualquer evento adverso, e recomendadas para não associar outros medicamen- tos naquele período sem notificação prévia. A média de idade foi de 24,5 $\pm 5,6$ anos; a paciente mais jovem tinha 17 anos e a mais idosa 36. Cerca de $19,3 \%$ das participantes eram adolescentes, 19,4\% tinham mais de 30 anos e mais de $60 \%$ estavam na terceira década.

Para a avaliação clínica consideraram-se os parâmetros idade, peso, estatura, pressão arterial, índice de massa corporal (IMC) e razão cinturaquadril. A cintura foi medida com a paciente em pé, na região mais estreita entre o arco da última costela e a crista ilíaca; a circunferência do quadril foi estimada pelo maior perímetro entre a cintura e a $\operatorname{coxa}^{7}$. Para o cálculo do IMC utilizou-se a fórmula IMC = peso $(\mathrm{kg}) /$ altura $^{2}(\mathrm{~m})$. O peso corporal médio foi de $55,4 \pm 7,1 \mathrm{~kg}$ e o IMC, restrito a mulheres com IMC abaixo de 26, foi de 21,6 $\pm 2,5$ $\mathrm{kg} / \mathrm{m}^{2}$. A razão cintura-quadril média foi de $0,79 \pm 0,06$, havendo $38 \%$ com perfil andróide e $62 \%$ ginecóide. Em relação à paridade, 38,7\% eram nuligestas, $41,9 \%$ primíparas, $16,2 \%$ secundíparas e $3,2 \%$ tercíparas.

FSH, LH, estradiol e PRL foram dosados entre o $18^{\circ}$ e o $21^{\circ}$ dia da tomada da pílula e no $7^{\circ}$ dia após a ingestão do último comprimido, dia de reiniciar nova cartela. Utilizou-se um preparado contendo $20 \mu \mathrm{g}$ de etinilestradiol e $75 \mu \mathrm{g}$ de gestodeno (Femiane ${ }^{\circledR}$, Schering do Brasil). Amostras de sangue foram colhidas pela manhã, após jejum de 8 a 12 horas, duas horas após o despertar e depois de repouso de 15 a 30 minutos no laboratório. Por punção da veia cubital colheramse $10 \mathrm{~mL}$ de sangue. Após repouso em temperatura ambiente por 60 minutos e formação de coágulo estável, a amostra foi centrifugada por 10 minutos a $1160 \mathrm{~g}$ (centrífuga modelo BE 404, série 2800, Bio Enge Indústria e Comércio Ltda., São Paulo). O soro foi transferido para tubos de polipropileno $(12 \times 125 \mathrm{~mm})$ e estocado a $-20^{\circ} \mathrm{C}$ até a realização dos exames. Todas as dosagens foram efetuadas no mesmo dia, com o mesmo kit de reagentes. Para a determinação da amostra consideraram-se variação máxima de $8,5 \%$ nos niveis de FSH e diferença significante mínima de $0,5 \mathrm{mUI} / \mathrm{mL}$ entre dois valores comparados, em análise de grupos pareados ${ }^{8}$. O estudo foi aprovado pelo Comitê de Ética em Pesquisa do Hospital Universitário Júlio Muller da Universidade Federal de Mato Grosso.

As dosagens hormonais foram efetuadas por quimioluminescência, utilizando-se kits Immulite $^{\circledR}$ obtidos da euro-DPC (Inglaterra), distribuídos no Brasil pela DPC Medlab Produtos Médico Hospitalares Ltda., São Paulo. Para dosagem do FSH os resultados, expressos em $\mathrm{mUI} / \mathrm{mL}$, foram padronizados de acordo com os termos da World 
Health Organization, Second International Reference Preparation (WHO, 2nd IRP 78/549) ${ }^{9}$. A concentração mínima detectável é de $0,1 \mathrm{mUI} / \mathrm{mL}$ e a máxima é de $170 \mathrm{mUI} / \mathrm{mL}$. Os coeficientes de variação intra-ensaios, com concentrações médias de 6,3, 16,0 e 45,3 mUI/mL, foram de 3,8, 6,2 e 5,3\%, respectivamente. Entre-ensaios, e nas concentrações de $6,9,17,1$ e 51,3 mUI/mL, as variações foram de $6,2,3,1$ e 3,5\%, respectivamente. O ensaio de LH foi padronizado pela WHO 1st IRP 68/ $40^{10}$, sendo os resultados também expressos em $\mathrm{mUI} / \mathrm{mL}$. A concentração mínima detectável é de 0,1 mUI/mL e o limite do ensaio é de $200 \mathrm{mUI} /$ mL. Os coeficientes de variação intra-ensaios nas concentrações médias de 9,7, 17,2 e 42,8 mUI/ $\mathrm{mL}$ foram de 4,8, 3,5 e 3,1\%, respectivamente. As variações entre-ensaios, para as concentrações de 10,9, 19,7 e 49,3 mUI/mL foram de 3,7, 4,3 e $3,3 \%$, respectivamente.

O estradiol foi dosado com o kit comercial Immulite ${ }^{\circledR}$, usando anticorpo monoclonal murino antiestradiol e anticorpo policlonal de cabra antiestradiol conjugado com fosfatase alcalina. Os resultados são expressos em $\mathrm{pg} / \mathrm{mL}$; a concentração mínima detectável é de $20 \mathrm{pg} / \mathrm{mL}$ e o limite do ensaio é de $200 \mathrm{pg} / \mathrm{mL}$. Os coeficientes de variação intra-ensaios com concentrações médias de 89, 229 e $1509 \mathrm{pg} / \mathrm{mL}$ foram de 7,0, 3,5 e 3,6\%, respectivamente. As variações entre-ensaios com concentrações de 95, 239 e $1532 \mathrm{pg} / \mathrm{mL}$ foram de $10,4,4,0$ e $5,6 \%$, respectivamente. Valores inferiores ao limite de detecção do ensaio foram considerados iguais a $20 \mathrm{pg} / \mathrm{mL}$.

O ensaio imunométrico utilizado para medir PRL foi também fornecido pela DPC, emprega dois anticorpos, um anticorpo monoclonal murino anti-PRL e um anticorpo policlonal de cabra antiPRL conjugado com fosfatase alcalina. Os resultados são expressos em ng/mL e padronizados de acordo com os termos do WHO 3rd IRP $84 / 500^{11}$. A faixa de calibração estende-se até a concentração de $150 \mathrm{ng} / \mathrm{mL}$. Os coeficientes de variação intra-ensaios com concentrações médias de 14,6, 21,3 e $64,0 \mathrm{ng} / \mathrm{mL}$, foram de $4,1,3,1$ e 3,3\%, respectivamente. As variações entre-ensaios, com concentrações de 14,4, 21,4 e 60,9 ng/mL foram de $4,1,2,4$ e $3,7 \%$, respectivamente.

Os parâmetros hormonais, descritos como média $(\bar{x})$ e erro padrão da média (EPM), foram comparados pelo teste $t$ de Student para amostras pareadas, utilizando o programa Epi-Info 6.04. A representação gráfica foi obtida com o programa STATA 6.0. O incremento nos níveis de FSH e LH, linearizados por transformação logarítmica, foi estimado pela fração b-f/b x 100, expressando a modificação percentual entre os valores basais e após a pausa ${ }^{12}$, onde $\mathrm{b}=$ valor basal e f $=$ valor após a pausa. A regressão linear foi utilizada para análise de associação entre os níveis dos diferentes hormônios e os parâmetros antropométricos. Esses dados foram expressos pelo coeficiente de correlação de Pearson $\left(\mathrm{r}^{2}\right)$ e analisados pelo teste $t$. As diferenças estatísticas foram consideradas significantes quando o valor de $\mathrm{p} \leq 0,05$.

\section{Resultados}

Cerca de $71 \%$ das pacientes estavam no primeiro ciclo de uso do anticoncepcional, 9,7\% faziam uso pelo segundo ciclo e as restantes $(19,3 \%)$ já eram usuárias de pílula por 3 a 48 meses. Em virtude do fato de que nem todas as pacientes foram avaliadas no primeiro ciclo, fezse inicialmente análise em separado dos níveis de FSH das pacientes já usuárias e das novas usuárias, comparando-se depois estes resultados com o verificado em toda a amostra. Este exercício não mostrou haver diferença significante entre os niveis de FSH das usuárias em primeiro ou segundo ciclo.

A média dos níveis de FSH entre o $18^{\circ}$ e o $21^{\circ}$ comprimido da cartela foi de $1,3 \pm 0,2$ e de $5,7 \pm 0,5 \mathrm{mUI} / \mathrm{mL}$ no $7^{\circ}$ dia de intervalo do contraceptivo. A média da diferença das concentrações de FSH nestes dois momentos foi de $4,4 \mathrm{mUI} / \mathrm{mL}$ (IC 95\% 3,4 a 5,4, $t=7,49$; p<0,05). O incremento observado nas concentrações de FSH entre os últimos comprimidos de uma cartela do contraceptivo e o dia do reinício do uso foi de $407 \%$ (Tabela 1). A média dos níveis de LH entre o $18^{\circ}$ e o $21^{\circ}$ dia da ingestão do comprimido da cartela foi de $0,8 \pm 0,1$ $\mathrm{mUI} / \mathrm{mL}$, e no $7^{\circ}$ dia do intervalo foi de $4,3 \pm 0,4$ $\mathrm{mUI} / \mathrm{mL}$, com incremento de $416 \%$ (Tabela 1). A concentração média do estradiol no dia da ingestão de um dos últimos comprimidos foi de $20,2 \pm 3,6$ e de $28,0 \pm 5,01 \mathrm{pg} / \mathrm{mL}$ no $7^{\circ}$ dia do intervalo do uso $(t=-6,39 ; p \leq 0,05)$. O incremento de estradiol observado na pausa foi de 39\%. A média dos níveis de PRL no dia da ingestão de um dos últimos comprimidos do contraceptivo foi de $12,4 \pm 1,3$ e de $9,1 \pm 0,9$ $\mathrm{ng} / \mathrm{mL}$ no $7^{\circ}$ dia de pausa $(t=0,885 ; \mathrm{p} \geq 0,05)$. A intensidade da diminuição da PRL, observada na pausa da pílula, foi de $26 \%$.

A análise de eventual associação entre as modificações do FSH nos dias de interrupção do anticoncepcional com o peso corpóreo não revelou qualquer associação entre estas variáveis, pelo menos nos indivíduos pesando até $70 \mathrm{~kg}\left(\mathrm{r}^{2}=0,090\right.$ e $b=-0,601 ; p=0,080$ ). Não se observou também associação entre o incremento de FSH na pausa 
da pílula com a razão cintura-quadril $\left(\mathrm{r}^{2}=0,003\right.$; $p=0,750)$ ou massa corporal $\left(r^{2}=0,030 ; p=0,290\right)$. $O$ incremento de $\mathrm{LH}$ foi menor nos indivíduos com peso maior que $55 \mathrm{~kg}$, sendo de $25 \%$ a influência do peso na atenuação dos níveis de LH (Figura 1), indicando que apenas $25 \%$ na variação do $\mathrm{LH}$ pode ser explicada pela variação do peso corporal.

Tabela 1 - Modificações nos níveis de FSH e LH durante o uso de pílula e após intervalo de sete dias.

\begin{tabular}{lcccc}
\hline & Final da cartela & $7^{0}$ dia de pausa & Diferença & Incremento (\%) \\
\hline $\mathrm{FSH}^{\mathrm{a}} \mathrm{mUl} / \mathrm{mL} \mathrm{x} \pm \mathrm{EPM}$ & $1,3 \pm 0,23$ & $5,7 \pm 0,55$ & $4,4 \pm 0,58$ & $2,6 \pm 0,18^{\mathrm{b}}$ \\
$\mathrm{LH}^{\mathrm{c}} \mathrm{mUl} / \mathrm{mL} \mathrm{x} \pm \mathrm{EPM}$ & $0,8 \pm 0,13$ & $4,4 \pm 0,44$ & $3,5 \pm 0,41$ & $2,6 \pm 0,14^{\mathrm{d}}$ \\
\hline
\end{tabular}

at $=7,49, p<0,05$ para FSH no final da cartela e no $7^{\circ}$ dia de pausa.

bMédia do incremento de $407 \%$.

ct $=8,68, p<0,05$ para LH no final da cartela e no $7^{\circ}$ dia de pausa.

dMédia do incremento de $416 \%$.

$\mathrm{X}=$ média

EPM = erro padrão da média

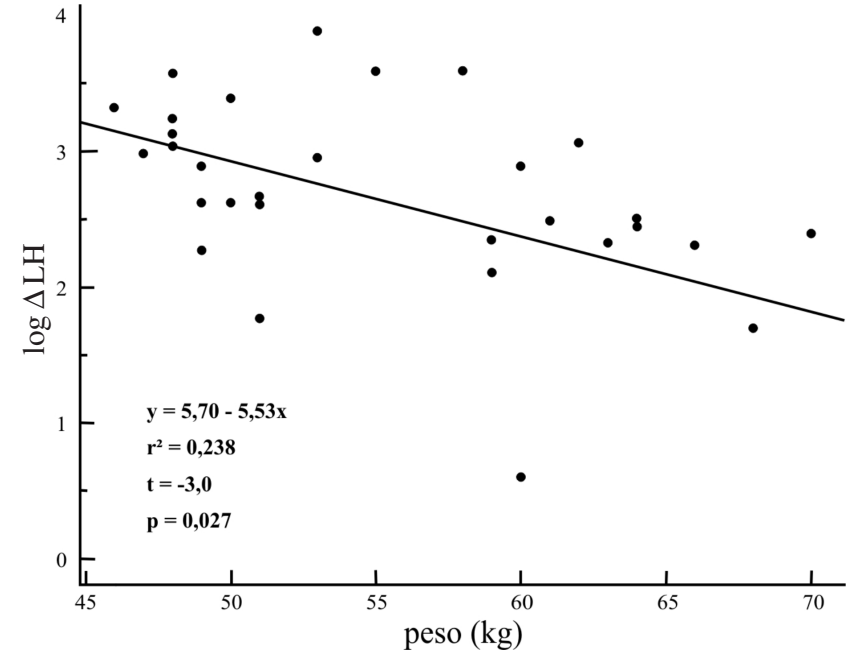

Figura 1 - Correlação entre o incremento nos níveis de LH na pausa da pílula e o peso corpóreo.

Houve fraca associação positiva entre elevação de LH na pausa da pílula com a massa corpórea $\left(r^{2}=0,152 ; p=0,030\right)$. Não houve associação entre incremento de LH e razão cintura-quadril $\left(\mathrm{r}^{2}=0,034\right.$; $\mathrm{p}=0,320$ ). Não se demonstrou também haver associação entre o aumento do estradiol na pausa do ACHO com qualquer parâmetro antropométrico, $r^{2}=0,070(p=0,149)$ para a associação estradiol e peso; $r^{2}=0,035(p=0,308)$ para a associação estradiol e IMC; $r^{2}=0,004(p=0,918)$ para a associação estradio, e razão cintura-quadril. Do mesmo modo, a diminuição da PRL não se associou com o peso corporal, $r^{2}=0,216(p=0,431) ; I M C, r^{2}=0,019(p=0,455)$ ou razão cintura-quadril, $\mathrm{r}^{2}=0,021(\mathrm{p}=0,427)$.

\section{Discussão}

A supressão das funções hipofisária e ova- riana é parcial quando se usam anticoncepcionais orais combinados, havendo pequeno crescimento dos folículos ovarianos durante a semana de pausa da pílula ${ }^{13}$. Diversos fatores controlam a atividade do eixo hipotálamo-hipofisário, incluindo a dose do estrogênio, o tipo e dose do progestagênio, o regime de administração e a resposta individual da usuária. Os efeitos inibitórios dos dois componentes são estabelecidos por interação sinérgica, tanto em nível hipotalâmico como hipofisário. Este estudo avaliou a influência da combinação do etinilestradiol, na dose de $20 \mu \mathrm{g}$, e do gestodeno, na de $75 \mu$ g por comprimido, em uso diário por 21 dias, com intervalo livre de 7 dias.

Como cerca de $80 \%$ das participantes tinham menos de 30 anos, a associação idade-função hipofisária não foi analisada neste estudo. De qualquer modo, a influência do fator idade na atenuação da função hipofisária pelos esteróides sexuais parece ocorrer apenas nas concentrações basais de FSH, sendo os níveis desta gonadotrofina mais elevados no último dia da pausa nas pacientes mais idosas ${ }^{14}$. A amostra incluída neste estudo era, na sua maioria, de primíparas $(41,9 \%)$ e nuligestas $(38,7 \%)$. Esta grande proporção de nuligestas e primíparas na amostra examinada não permitiu analisar o papel da paridade sobre a resposta hipotálamo-hipofisária durante o uso do anticoncepcional.

Na semana do intervalo livre da medicação, os níveis de FSH sofreram incremento superior a quatro vezes. Níveis de FSH menores que $1 \mathrm{mUI} /$ mL durante uso de ACHO, indicando forte atenuação da função hipotálamo-hipofisária, já tinham sido observados por Crosignani et al. ${ }^{15}$, empregando a mesma combinação do presente estudo, e van Heusden e Fauser ${ }^{16}$, com a combinação de $30 \mu \mathrm{g}$ de etinilestradiol e $150 \mu \mathrm{g}$ de desogestrel. No estudo destes dois últimos autores, usando combi- 
nação mais estrogênica, o incremento de FSH durante a pausa foi maior. Com a combinação de $20 \mu \mathrm{g}$ de etinilestradiol e $75 \mu \mathrm{g}$ de desogestrel, Hemrika et al. ${ }^{17}$ observaram concentração de FSH menor que $1 \mathrm{mUI} / \mathrm{mL}$ durante o uso e recuperação de dez vezes no $7^{\circ}$ dia de pausa.

Em conjunto, estas observações demonstram, confirmando estudos anteriores, a ocorrência de forte atenuação do eixo hipotálamohipofisário, resultando em concentrações de FSH compativeis com níveis pré-puberais, e não com os observados na fase folicular precoce como usualmente descrito. Algumas publicações têm sugerido que gestodeno e desogestrel têm maior efeito inibitório sobre o FSH do que o levonorgestrel ${ }^{16}$. No entanto, estudos bem controlados indicam que o grau de atenuação depende mais da dose do que do tipo do componente progestagênico ${ }^{18}$, aceitando-se que a nova geração destes produtos seja mais eficiente para o bloqueio das gonadotrofinas que os preparados mais antigos. A pequena diferença no grau de atenuação do FSH observada entre o presente estudo e publicações anteriores ${ }^{16,17}$ pode ser devida aos diferentes ensaios utilizados. Enquanto empregou-se a quimioluminescência no presente estudo, os dois maiores estudos usados para comparação utilizaram ensaios radioimunométricos. Aceita-se que dosagens hormonais com métodos radioimunométricos apresentem concentrações até $50 \%$ maiores do que as obtidas com ensaios imunoenzimáticos ${ }^{19}$.

Os níveis de gonadotrofinas ao término da cartela do ACHO atenderam ao critério de supressão proposto por Rossmanith et al. ${ }^{20}$ ( $\mathrm{LH}$ inferior a 1,5 mUI/mL e FSH menor que $2 \mathrm{mUI} / \mathrm{mL}$ ) em $87 \%$ dos ciclos. Com uma combinação contendo $20 \mu \mathrm{g}$ de etinilestradiol e 0,5 mg de noretisterona, estes autores relataram supressão acentuada de LH em $76 \%$ dos ciclos de pílula e supressão parcial no restante. Em estudo no qual avaliaram-se dois preparados contendo $20 \mu \mathrm{g}$ de etinilestradiol com $75 \mu \mathrm{g}$ ou $150 \mu \mathrm{g}$ de desogestrel, os níveis de LH foram suprimidos a 0,7 e 0,2 mUI/mL, mostrando a influência da dose do progestagênio no bloqueio da secreção do $\mathrm{LH}^{16}$. O estudo de Hemrika et al. ${ }^{17}$, avaliando dois preparados com $30 \mathrm{vg}$ de etinilestradiol e dois diferentes progestagênios (levonorgestrel e desogestrel) na mesma dosagem (150 $\mu \mathrm{g})$, mostrou o mesmo grau de atenuação dos niveis basais de LH e FSH.

No presente estudo, os níveis de LH também sofreram um incremento de pelo menos quatro vezes durante a pausa da combinação examinada, indicando recuperação da hipófise com a mesma magnitude que a observada para o FSH. No estudo de Hemrika et al. ${ }^{17}$, o incremento do LH foi de sete vezes. No entanto, este maior aumento não está claro, já que a população tinha a mesma faixa etária e as amostras foram colhidas também no sétimo dia de pausa e entre o $13^{\circ}$ e o $26^{\circ}$ dia de uso. Como van Heusden e Fauser ${ }^{16}$ observaram recuperação do LH da ordem de 25 vezes e a população analisada também pertencia à mesma faixa etária, podese especular que levonorgestrel e desogestrel tenham potências diferentes na modulação do LH.

Os níveis de estradiol foram inferiores ao do limite de detecção do ensaio em todas as usuárias ao término da cartela, denotando bloqueio acentuado da esteroidogênese folicular. A elevação de 40\% observada nos níveis de estradiol no período livre de pílula indica reinício precoce da função das células teca-granulosas. Tem-se observado que no final desta recuperação os níveis de estradiol permanecem 50-60\% menores que os observados na fase folicular precoce de não usuárias normais ${ }^{21}$. van Heusden e Fauser ${ }^{16}$ identificaram incremento de aproximadamente quatro vezes no nível sérico de estradiol na pausa da pílula, mas a recuperação ocorrida no presente estudo, menor que a relatada por aqueles autores, não parece ser devida somente aos diferentes progestagênios utilizados. É possível que se deva, em parte, também aos diferentes ensaios empregados nos dois estudos. A nãoocorrência de elevação do estradiol acima do limite de detecção do ensaio em quase metade das usuárias sugere que o bloqueio ovariano ainda permanece acentuado nos dias de pausa da pílula em número significativo de mulheres.

Ainda que Jernstrom et $a .^{22}$ não tenham encontrado nenhuma diferença nos níveis de PRL entre usuárias, ex-usuárias e não usuárias de pílula, observou-se, no presente estudo, decréscimo de $26 \%$ nas concentrações deste hormônio durante a semana de pausa. Essa discrepância pode ser atribuída às diferentes técnicas utilizadas para a dosagem da PRL (quimioluminescência $v$ s radioimunoensaio). De modo geral, durante o uso do ACHO, tem-se observado aumento de PRL proporcional à dose do componente estrogênico do produto utilizado ${ }^{23}$. O presente estudo não fez esta avaliação, direcionando seu objetivo para a modificação na pausa e não durante os 21 dias de uso da pílula.

São escassos os dados acerca da influência das variáveis antropométricas sobre os efeitos do ACHO em nivel hipotalâmico-hipofisário. No grupo de mulheres por nós estudadas o IMC não ultrapassou 25, por atender a critério de inclusão. Parece que, em usuárias de pílulas contendo entre 30$35 \mu \mathrm{g}$ de etinilestradiol e progestagênios menos androgênicos, o IMC também não influencia os níveis de FSH, mas os niveis de $\mathrm{LH}$ parecem sofrer diminuição com maior intensidade em usuárias 
magras $^{24}$. Com IMC médio de 21,6 percebeu-se haver associação negativa entre peso corporal e incremento de $\mathrm{LH}$ no período de pausa da pílula. No entanto, o incremento dos níveis de FSH não mostrou qualquer associação com essas duas variáveis. Ainda que o IMC tenha sido limitado a 25 nessa amostra, a razão cintura-quadril foi maior que 0,8 em quase 39\% dela. Os incrementos observados nos níveis de FSH e LH não mostraram associação com essa variável.

Este estudo demonstrou acentuado grau de atenuação das gonadotrofinas hipofisárias durante o uso do anticoncepcional e rápido poder de recuperação hipotálamo-hipofisário no intervalo de sete dias entre duas cartelas de pílula. Esse fato indica rápido início da folículogênese e pode ser importante para o clínico na identificação da reserva ovariana em usuárias de contraceptivo hormonal oral, principalmente naquelas com mais de 35 anos de idade.

\section{ABSTRACT}

Objective: to evaluate serum levels of follicle-stimulating hormone (FSH) and luteinizing hormone (LH) in the pillfree interval of a combined oral contraceptive containing 20 $\mu \mathrm{g}$ of ethynylestradiol and $75 \mathrm{mg}$ of gestodene.

Methods: thirty-one women from 17 to 36 years old, mean age of 24.5 years old, $19 \%$ adolescents, were included. FSH, $L H$, prolactin $(P R L)$ and estradiol $\left(E_{2}\right)$ levels were measured by immunochemoluminescence. Both FSH and LH levels were measured within the last four days of pill intake and on the 7 th day of the pill-free interval between two cycles. Hormonal levels were compared by the Student t-test. Comparisons between hormonal and anthropometric data were made by linear regression; values of $p<0.05$ were taken as significant. Results: seventy-one percent of women were using the pill for the first time. FSH levels increased from 1.3 to $5.7 \mathrm{mIU} / \mathrm{ml}$ between the end of the blister pack and the 7th day of the pillfree interval. LH increased from 0.8 to $4.3 \mathrm{mIU} / \mathrm{ml}$. E $E_{2}$ levels changed from 20.2 to $28.0 \mathrm{pg} / \mathrm{ml}$. The levels of PRL decreased from 12.4 to $10.2 \mathrm{ng} / \mathrm{ml}$. There was no correlation between the changes in gonadotrophin levels and most of the anthropometric parameters in these women, with body mass index $<25 \mathrm{~kg} / \mathrm{m}^{2}$. Conclusion: the gonadotrophin levels detected on the last four days of pill intake were greatly suppressed, recovery of three to four times in amount occurring on the 7th day of the pill-free interval.

KEYWORDS: Oral contraceptive. Gonadotrophins. Prolactin.

\section{Agradecimentos}

Os autores são gratos aos Laboratórios Schering do Brasil, por prover gratuitamente anticoncepcional às pacientes incluídas no estudo.

\section{Referências}

1. Moore RY. Mecanismos neuroendócrinos: células e sistemas. In: Yen SC, Taffe RB, editores. Endocrinologia Reprodutiva. Fisiologia, fisiopatologia e tratamento clínico. $2^{\mathrm{a}}$ ed. São Paulo: Roca; 1990. p. 3-27.

2. Menon M, Peegel H, Katta V. Estradiol potentiation of gonadotropin-releasing hormone responsiveness in the anterior pituitary is mediated by an increase in gonadrotopin-releasing hormone receptors. Am J Obstet Gynecol 1985; 151:534-40.

3. Speroff L, Glass RH, Kase, NG. Endocrinologia ginecológica clínica e infertilidade. $4^{\underline{a}}$ ed. São Paulo: Manole; 1991. p. 544.

4. Dericks-Tan JS, Gudacker V, Taubert HD. Influence of oral contraceptives on integrated secretion of gonadotropins. Contraception 1992; 46:369-77.

5. Soules MR, Steiner RA, Clifton OK, Cohen NL, Aksel $\mathrm{S}$, Bremner WJ. Progesterone modulation of pulsatile luteinizing hormone secretion in normal women. J Clin Endocrinol Metab 1984; 58:378-83.

6. Henzl MR. Contraceptive hormones and their clinical use. In: Yen SSC, Jaffe RB, editors. Reproductive Endocrinology. Physiology, pathophysiology, and clinical management. $1^{\text {st }}$ ed. Philadelphia: WB Saunders; 1986. p. 643-82.

7. Kamimura MA, Baxmann A, Sampaio LR, Cuppari L. Avaliação nutricional. In: Cuppari L, editora. Nutrição. Nutrição clínica no adulto. $1^{\mathrm{a}}$ ed. São Paulo: Manole; 2002. p. 71-109.

8. Kirby A, Gebski V, Keech AC. Determining the sample size in a clinical trial. Med J Aust 2002; 177:256-7.

9. Rose MP. Follicle stimulating hormone: international standars and reference preparations for the calibration of immunoassays and bioassays. Clin Chim Acta 1998; 273:103-17.

10.Storring PL, Zaidi AA, Mistry YG, Lindberg M, Stenning BE, Diczfalusy E. A comparison of preparations of highly purified human pituitary luteinizing hormone: differences in the luteinizing hormone potencies as determined by in-vivo bioassay, in vitro bioassay and immunoassay. Acta Endocrinol (Copenh) 1982; 101:339-47.

11. World Health Organization. International biological reference preparation: catalogue. Geneva; 2001.

12. Matthews JN, Altman DG, Campbell MJ, Royston P. Analysis of serial measurements in medical research. BMJ 1990; 300:230-5.

13.Schlaff WD, Lynch AM Hughes HD, Cedars MI, Smith DL. Manipulation of the pill-free interval in oral contraceptive users: the effect on follicular supression. Am J Obstet Gynecol 2004; 190:943-51. 
14.Fitzgerald C, Elstein M, Spona J. Effect of age on the response of the hypothalamo-pituitary-ovarian axis to a combined oral contraceptive. Fertil Steril 1999; 71:1079-83.

15. Crosignani PG, Testa G, Vegetti W, Parazzini F. Ovarian activity during regular oral contraceptive use. Contraception 1996; 54:271-3.

16.Van Heusden AM, Fauser BC. Activity of the pituitary-ovarian axis in the pill-free interval during use of low-dose combined oral contraceptives. Contraception 1999; 59:237-43.

17.Hemrika DJ, Slaats EH, Kennedy JC, de Vries Robles-Korsen TJ, Schoemaker J. Pulsatile luteinizing hormone patterns in long term oral contraceptive users. J Clin Endocrinol Metab 1993; 77:420-6.

18. Grimes DA, Godwin AJ, Rubin A, Smith JA, Lacarra M. Ovulation and follicular development associated with three low-dose oral contraceptvies: a randomized controlled trial. Obstet Gynecol 1994; 83:29-34.

19. Olivares A, Cárdenas M, Timossi C, Zarinan T, DíazSanchez V, Ulloa-Aguirre A. Reactivity of different LH and FSH standards and preparations in the World Health Organization matched reagents for enzyme-linked immunoassays of gonadotrophins. Hum Reprod 2000; 15:2285-91.
20.Rossmanith WG, Steffens D, Schramm G. A comparative randomized trial on the impact of two lowdose oral contraceptives on ovarian activity, cervical permeability, and endometrial receptivity. Contraception 1997; 56:23-30.

21.Van der Spuy ZM, Sohnius U, Pienaar CA, Schall R. Gonadotropin and estradiol secretion during the week of placebo therapy in oral contraceptive pill users. Contraception 1990; 42:597-609.

22.Jernstrom H, Knutsson M, Taskila P, Olsson H. Plasma prolactin in relation to menstrual cycles phase, oral contraceptive use, arousal time and smoking habits. Contraception 1992; 46:543-8.

23.Ismail MS, Serour GI, Torsten U, Weitzel H, Berlien HP. Elevated serum prolactin level with high-dose estrogen contraceptive pills. Eur J Contracept Reprod Health Care 1998; 3:45-50.

24. Cibula D, Hill M, Fanta M, Sindelka G, Zivny J. Does obesity diminish the positive effect of oral contraceptive treatment on hyperandrogenism in women with polycystic ovarian syndrome? Hum Reprod 2001; 16:940-4. 